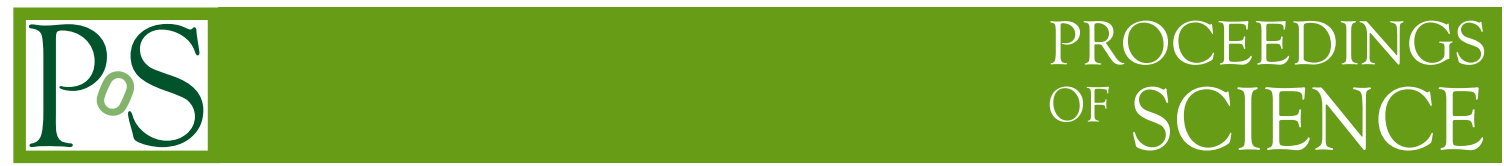

\title{
Superintegrable systems related to truncated Calogero model
}

\section{S. Vardanyan* and T. Hakobyan}

Yerevan State University,

1 Alex Manoogian Street, Yerevan, 0025, Armenia

E-mail: sedavardanyan5@gmail.com, tigran.hakobyan@ysu.am

A simple $U(1)$ gauge transformation, which is equivalent to the inclusion of the Aharonov-Bohm type potential, is introduced and applied to superintegrable systems in classical and quantum mechanics. It produces an inverse-square potential with two- and three-particle interactions which is identical to the potential of the recently studied truncated Calogero model.

RDP online workshop "Recent Advances in Mathematical Physics" - Regio2020,

5-6 December 2020

online

${ }^{*}$ Speaker 


\section{Introduction}

The $N$-dimensional free particle, isotropic oscillator and particle moving in the Coulomb potential are the simplest maximally superintegrable systems. Apart from the Liouville integrals, such systems have $N-1$ additional constants of motion. Due to them, the orbits of the classical bounded motion are closed. For quantum systems, the superintegrability leads to a hight degeneracy of energy levels and an exact expression for the wavefunctions. The entire symmetry of the free-particle, oscillator and Coulomb systems constitute, correspondingly, the Euclidean $E(N)$, unitary $U(N)$ and (pseudo)orthogonal $S O(N+1) / S O(1, N)$ groups, which are responsible for the superintegrability. They all contain the $S O(N)$ rotational subgroup, which is the symmetry of the angular part of the above Hamiltonians. The described models lie at the origin of many (super)integrable models of classical and quantum mechanics.

Treating each coordinate as a separate particle in one dimension, one can turn on an interaction between them. In particular, the inverse-square potential, introduced by Calogero, significantly complicates the above systems [1-5]. Nevertheless, it preserves the superintegrability [6-9]. In the quantum case, the Calogero potential can be involved into a covariant derivative with flat connection, bringin closer to the original system [10, 11]. It was first introduced by Dunkl and contains particle-exchange operators [12]. As a result, the Calogero model (unbound or bound by oscillator or Coulomb potential) can be regarded as a Dunkl-operator deformation of the underlying system without particle interaction. The related symmetries are deformed as well. Their generators together with the exchange operators form a quadratic algebra [9, 13-15].

Moreover a nonlocal gauge (similarity) transformation eliminates the connection together with the Calogero potential [16]. For indistinguishable bosons or fermions, it just shifts the ground-state energy level in the bound spectrum ensuring an equivalence with the related noninteracting system. Recently, a modified analog of this transformation have been applied in order to construct Calogerotype model with more general inverse-square interaction, containing also three-particle terms [17]. In contrast to the pure Calogero case, here most wavefunctions of the noninteracting oscillators are mapped to non-normalizable states. In the current talk, we introduce a simpler $U(1)$ gauge transform in $N$-dimensional space, which produce not only similar-type inverse-square potentials but also but also terms linear in momentum. It is equivalent to inclusion of the Aharonov-Both type magnetic potential which is inverse in coordinates. For quantum case, the momentum shift is reminiscent the truncated Dunkl operator without the particle exchanges. For an imaginary phase, the map produces non-Hermitian $P T$-invariant Hamiltonians.

\section{Canonical mapping of classical Hamiltonians}

Consider the following transformation of the phase space variables of an $\mathrm{N}$-dimensional system, which mixes the coordinate and momentum:

$$
p_{i}^{\prime}=p_{i}+\sum_{1 \leq|i-j| \leq r} \frac{f}{x_{i}-x_{j}}, \quad x_{i}^{\prime}=x_{i} .
$$


It depends on an integral parameter $r \leq N-1$. For the classical systems, the above relation describes a canonical map with singularities at the hyperplanes $x_{i}=x_{j}$ with $|i-j| \leq r$ :

$$
\sum_{i=1}^{N} p_{i}^{\prime} d x_{i}^{\prime}=\sum_{i=1}^{N} p_{i} d x_{i}+d \log F^{f}, \quad F=\prod_{1 \leq i-j \leq r}\left|x_{i}-x_{j}\right|
$$

so that the new variables obey the standard Poisson brackets,

$$
\left\{p_{i}^{\prime}, x_{j}\right\}=\delta_{i j}, \quad\left\{p_{i}^{\prime}, p_{j}^{\prime}\right\}=0
$$

Note that the primed momentum can be interpreted as a generalized momentum in the Aharonov-Bohm like magnetic potential

$$
A_{i}=\partial_{i} \log F^{f}
$$

First, let us consider the free-particle system. It is easy to see that the above transformation maps it to an equivalent model with the two-particle and three-particle distant interactions,

$$
H=\sum_{i=1}^{N} \frac{p_{i}^{\prime 2}}{2}=\sum_{i=1}^{N} \frac{p_{i}^{2}}{2}+\sum_{1 \leq i-j \leq r}\left(\frac{f^{2}}{\left(x_{i}-x_{j}\right)^{2}}+f \frac{p_{i}-p_{j}}{x_{i}-x_{j}}\right)-\sum_{\substack{i<j<k \\ r<k-i \leq 2 r}} \frac{f^{2}}{\left(x_{i}-x_{j}\right)\left(x_{j}-x_{k}\right)}
$$

Of course, the above system is superintegrable with integrals of motion given by the momentum $p_{i}^{\prime}$ and angular momentum $L_{i j}^{\prime}=x_{i} p_{j}^{\prime}-x_{j} p_{i}^{\prime}$ with the standard Poisson brackets between them.

It is well known that both the isotropic harmonic and Coulomb potentials preserve the superintegrability. Apply the following notations for the corresponding Hamiltonians:

$$
H_{\omega}=H+\frac{\omega^{2} r^{2}}{2}, \quad H_{\gamma}=H-\frac{\gamma}{r}, \quad r^{2}=\sum_{i} x_{i}^{2} .
$$

The integrals of motion in the first case form the $u(N)$ Lie algebra with the angular momentum and Fradkin tensor $T_{i j}^{\prime}=x_{i} x_{j}+p_{i}^{\prime} p_{j}^{\prime}$. In the Coulomb case, the symmetry forms the $s o(4)$ or $s o(1,3)$ Lie algebra (depending on whether the energy is negative or positive). The generators are expressed via the angular momentum, Hamiltonian $H_{\gamma}$, and the Runge-Lenz vector $A_{i}^{\prime}=\sum_{j} x_{j} L_{i j}^{\prime}-\gamma x_{i} / r$.

Of course, the canonical map (1) may be applied to other models. The obtained Hamiltonians look quite close to the rational Calogero model, which describes the motion of one-dimensional particles with pairwise inverse-square interaction [1,2]. The Calogero model with and without the oscillator or Coulomb potentials, is maximally superintegrable [6-9]. The shifted version is governed by the following Hamiltonian:

$$
\begin{aligned}
H_{g}= & \sum_{i} \frac{p_{i}^{\prime 2}}{2}+\sum_{i<j} \frac{g^{2}}{\left(x_{i}-x_{j}\right)^{2}}=\sum_{i} \frac{p_{i}^{2}}{2}+\sum_{1 \leq i-j \leq r}\left(\frac{f^{2}+g^{2}}{\left(x_{i}-x_{j}\right)^{2}}+f \frac{p_{i}-p_{j}}{x_{i}-x_{j}}\right) \\
& +\sum_{i-j>r} \frac{g^{2}}{\left(x_{i}-x_{j}\right)^{2}}-\sum_{\substack{i<j<k \\
r<k-i \leq 2 r}} \frac{f^{2}}{\left(x_{i}-x_{j}\right)\left(x_{j}-x_{k}\right)} .
\end{aligned}
$$


Note that in $r=N-1$ case, the last two terms in the Hamiltonian (6) disappear, and the system becomes invariant under particles exchanges.

Clearly, the constructed model is superintegrable too. The Lax matrix is inherited from the Calogero model [2]

$$
\mathcal{L}_{i j}=\delta_{i j} p_{i}^{\prime}+\left(1-\delta_{i j}\right) \frac{\imath g}{x_{i}-x_{j}}
$$

where the $p_{i}^{\prime}$ is defined by (1). It defines the Liouville integrals of motion $I_{n}=\operatorname{Tr} \mathcal{L}^{n}$ for $n \leq N$. The additional integrals may be defined by taking their Poisson brackets with the angular part of the Hamiltonian $H_{g}$ alike in non-deformed $(f=0)$ case [19].

Note that the Hamiltonian (6) is reminiscent of the classical version of the truncated Calogero model without external harmonic potential. The later contains both the two-body and three-body inverse-square interactions with restricted interaction distance between particles [17]. Let us apply the canonical transformation (1) to the truncated Calogero model with the particular choice for restriction parameter equal to $r$,

$$
\begin{aligned}
H_{g, r} & =\sum_{i=1}^{N} \frac{p_{i}^{\prime 2}}{2}+\sum_{1 \leq i-j \leq r} \frac{g^{2}}{\left(x_{i}-x_{j}\right)^{2}}-\sum_{\substack{i<j<k \\
r<k-i \leq 2 r}} \frac{g^{2}}{\left(x_{i}-x_{j}\right)\left(x_{j}-x_{k}\right)} \\
& =\sum_{i=1}^{N} \frac{p_{i}^{2}}{2}+\sum_{1 \leq i-j \leq r}\left(\frac{f^{2}+g^{2}}{\left(x_{i}-x_{j}\right)^{2}}+f \frac{p_{i}-p_{j}}{x_{i}-x_{j}}\right)-\sum_{\substack{i<j<k \\
r<k-i \leq 2 r}} \frac{f^{2}+g^{2}}{\left(x_{i}-x_{j}\right)\left(x_{j}-x_{k}\right)} .
\end{aligned}
$$

\section{Gauge transformation of quantum systems}

In the quantum case, the canonical map (1), (2) is generated by the following gauge transformation acting on any function $G$ depending on the phase space variables:

$$
G\left(p_{i}, x_{i}\right) \rightarrow U^{-1} G\left(p_{i}, x_{i}\right) U=G\left(p_{i}^{\prime}, x_{i}\right)
$$

with $p_{i}=-\imath \partial_{i}$. Here the local $U(1)$ phase

$$
U=F^{l f}=\exp \left(\text { lf } \sum_{1 \leq i-j \leq r} \ln \left|x_{i}-x_{j}\right|\right)
$$

defines an unitary shift which does not contain any singularity.

In particular, the free particle is mapped to the following system:

$$
\begin{aligned}
H & =U^{+}\left(\sum_{i=1}^{N} \frac{p_{i}^{2}}{2}\right) U=\sum_{i=1}^{N} \frac{p_{i}^{\prime 2}}{2} \\
& =\sum_{i=1}^{N} \frac{p_{i}^{2}}{2}+\sum_{1 \leq i-j \leq r}\left(\frac{f(f+\imath)}{\left(x_{i}-x_{j}\right)^{2}}+\frac{f}{x_{i}-x_{j}}\left(p_{i}-p_{j}\right)\right)-\sum_{\substack{i<j<k \\
r<k-i \leq 2 r}} \frac{f^{2}}{\left(x_{j}-x_{i}\right)\left(x_{i}-x_{k}\right)} .
\end{aligned}
$$


Note that the quantum corrections make the coefficient in front of the inverse-square potential a complex number [compare with (4)]. The classical coefficient recovers upon applying the anticommutator in the momentum term:

$$
H=\sum_{i=1}^{N} \frac{p_{i}^{2}}{2}+\sum_{1 \leq i-j \leq r}\left(\frac{f^{2}}{\left(x_{i}-x_{j}\right)^{2}}+\left\{\frac{f}{x_{i}-x_{j}}, \frac{p_{i}-p_{j}}{2}\right\}\right)-\sum_{\substack{i<j<k \\ r<k-i \leq 2 r}} \frac{f^{2}}{\left(x_{j}-x_{i}\right)\left(x_{i}-x_{k}\right)} .
$$

The Hermiticity of the Hamiltonian becomes transparent in this form.

Obviously, the unitary map (8) does not change the spectrum. It just produces the local phase factor in front of the wavefunctions. For the usual plane waves, we have:

$$
\psi_{k_{1} \ldots k_{N}}(x)=e^{i \sum_{i} k_{i} x_{i}-l f \sum_{1 \leq i-j \leq r} \ln \left|x_{i}-x_{j}\right|}
$$

with continuous momenta $k_{i}$ defining the spectrum,

$$
E_{k_{1} \ldots k_{N}}=\sum_{i=1}^{N} \frac{k_{i}^{2}}{2}
$$

Consider the system bound by the harmonic potential $H_{\omega}(5)$ with unit frequency, $\omega=1$. For $r=1$, when there are only the nearest-neighbouring interactions, this system was already studied [20]. Recalling the energy eigenfunctions of the usual isotropic oscillator, one can immediately write those for the current Hamiltonian (without the normalization constant),

$$
\psi_{k_{1} \ldots k_{N}}(x)=e^{-\frac{1}{2} r^{2}-l f \sum_{1 \leq i-j \leq r} \ln \left|x_{i}-x_{j}\right|} \prod_{i=1}^{N} H_{k_{i}}\left(x_{i}\right),
$$

where $k_{i} \geq 0$ are integral numbers, and the $H_{k}(x)$ is the $k$-th order Hermite polynomial. The corresponding eigenvalues are the same as that of the free oscillators:

$$
E_{k_{1} \ldots k_{N}}=\sum_{i=1}^{N} k_{i}+\frac{N}{2}
$$

For the bound motion in the Coulomb potential (5) with unit constant, $\gamma=-1$, the energy spectrum is quantized thought the principal quantum number $n$,

$$
E_{n}=-\frac{1}{2 n^{2}}, \quad \text { with } \quad n=n_{r}+l+\frac{N-1}{2},
$$

where $n_{r}, l=0,1, \ldots$ are the radial and angular momentum quantum numbers, respectively. The wavefunctions (without normalization) are expressed via the associated Laguerre polynomials:

$$
\psi_{n l}(x)=e^{-\frac{1}{n} r-l f \sum_{1 \leq i-j \leq r} \ln \left|x_{i}-x_{j}\right|} L_{n_{r}}^{2 l+N-2}\left(\frac{2 r}{n}\right) h_{l}(x) .
$$

Here the $h_{l}(x)$ is an homogeneous harmonic polynomial of degree $l$, which is proportional to the hyperspherical harmonics. Apart from the angular momentum, it depends also on other $N-$ 2 quantum numbers which are not indexed here. Such numbers characterize the states of the corresponding $S O(N)$ representation with angular momentum $l$,

$$
L^{2} h_{l}(x)=\sum_{i<j} L_{i j}^{2} h_{l}(x)=l(l+N-2) h_{l}(x) .
$$




\section{Shifted quantum Calogero model}

For indistinguishable particles, the following parametrization for the coupling constant is more convenient for the quantum Calogero model (6),

$$
H_{g}=\sum_{i=1}^{N} \frac{p_{i}^{\prime 2}}{2}+\sum_{i<j} \frac{g(g \mp \hbar)}{\left(x_{i}-x_{j}\right)^{2}} .
$$

The (+) and (-) sign is set for the fermions and bosons, respectively. The Plank's constant is recovered here in order to match with the classical case (15). In the following, we will set it to unity again $(\hbar=1)$ and consider solely the the bosonic particles with the minus sign in the potential.

The explicit form of the Hamiltonian follows immediately from the expression (10),

$$
\begin{aligned}
H_{g}= & \sum_{i} \frac{p_{i}^{2}}{2}+\sum_{1 \leq i-j \leq r}\left(\frac{f(f+\imath)+g(g-1)}{\left(x_{i}-x_{j}\right)^{2}}+\frac{f}{x_{i}-x_{j}}\left(p_{i}-p_{j}\right)\right)+\sum_{i-j>r} \frac{g(g-1)}{\left(x_{i}-x_{j}\right)^{2}} \\
& -\sum_{\substack{i<j<k \\
r<k-i \leq 2 r}} \frac{f^{2}}{\left(x_{i}-x_{j}\right)\left(x_{j}-x_{k}\right)} .
\end{aligned}
$$

The above Hamiltonian is closely related to another model usually referred as a generalized Calogero model with the particle exchanges involved in the potential [10,11]. Applying the unitary shift (9) to the generalized Calogero model itself, we get:

$$
\tilde{H}_{g}=\sum_{i=1}^{N} \frac{\pi_{i}^{2}}{2}=\sum_{i=1}^{N} \frac{p_{i}^{\prime 2}}{2}+\sum_{i<j} \frac{g\left(g-M_{i j}^{\prime}\right)}{\left(x_{i}-x_{j}\right)^{2}}, \quad M_{i j}^{\prime}=U^{+} M_{i j} U .
$$

Here the exchange operator $M_{i j}$ permutes the coordinates of the $i$-th and $j$-th particles. For indistinguishable particles, $M_{i j}= \pm 1$, depending on whether they are bosons or fermions, and the above Hamiltonian is reduced to the usual Calogero model (15). The deformed momentum is defined through the covariant derivative, expressed in terms of the shifted Dunkl operator [12],

$$
\nabla_{i}=\partial_{i}+\sum_{j \neq i} \frac{\imath f}{x_{i}-x_{j}}-\sum_{j \neq i} \frac{g}{x_{i}-x_{j}} M_{i j}^{\prime}, \quad \pi_{i}=-\imath \nabla_{i} .
$$

Remember that the Dunkl operators obey a deformed Heisenberg algebra relations, which, of course, are not affected by the gauge transformation $U$,

$$
\left[\nabla_{i}, \nabla_{j}\right]=0, \quad\left[\nabla_{i}, x_{j}\right]=S_{i j} .
$$

Here the symmetric matrix $S_{i j}$ depends of the particle-permutation operators,

$$
S_{i j}=\left(\delta_{i j}-1\right) g M_{i j}^{\prime}+\delta_{i j}\left(1+g \sum_{k \neq i} M_{i k}^{\prime}\right) .
$$

Notice that at a first glance, the flat connection in the covariant derivative can be eliminated by the gauge transformation mapping the model (17) to the free particles. However, this map is complicate due to the exchange terms. For the Hamiltonian (15) in the harmonic potential this results in an overall shift of the energy spectrum [21]. 
The Calogero model with particle exchanges (17) and its oscillator $\tilde{H}_{g, \omega}$ and Coulomb $\tilde{H}_{g, \gamma}$ versions (5) are superintegrable. The corresponding integrals of motion are reminiscent of those for the free particle, isotropic oscillator and Coulomb system, correspondingly. The integrals of motion are obtained by substituting the usual momentum by the Dunkl momentum: $p_{i}^{\prime} \rightarrow \pi_{i}$, see, for example, $[8,10,11,13,14,18]$ for the non-shifted case $(f=0)$.

Symmetric polynomials in such Dunkl-deformed integrals produce the constants of motion for the pure Calogero model (15), (16). In particular, the Newton powers

$$
\sum_{i=1}^{N} \pi_{i}^{n}, \quad \sum_{i=1}^{N}\left(a_{i}^{+} a_{i}\right)^{n}
$$

with $n \leq N$ reproduces the complete set of Liouville integrals of the Hamiltonians $H_{g}$ and $H_{g, \omega}$, respectively [10]. Here the $a_{i}^{ \pm}$are the staircase operators, which generate the spectrum of the Calogero-oscillator Hamiltonian with particle exchanges:

$$
a_{i}^{ \pm}=\sqrt{\frac{\omega}{2}} x_{i} \mp \frac{\nabla_{i}}{\sqrt{2 \omega}}, \quad H_{g, \omega}=\frac{\omega}{2} \sum_{i=1}^{N}\left(a_{i}^{+} a_{i}+a_{i} a_{i}^{+}\right), \quad\left[H_{g, \omega}, a_{i}^{ \pm}\right]= \pm \omega a_{i}^{ \pm} .
$$

The eigenstates of the Calogero model are restricted to the symmetric functions on particle coordinates (to the antisymmetric ones, for fermions) [1]. In the Calogero-oscillator case $H_{g, \omega=1}$, they may be written as follows:

$$
\psi_{k_{1} \ldots k_{N}}(x)=e^{-l f \sum_{1 \leq i-j \leq r} \ln \left|x_{i}-x_{j}\right|} A_{1}^{k_{1}} A_{2}^{k_{2}} \ldots A_{N}^{k_{N}} e^{-\frac{1}{2} r^{2}}, \quad A_{l}=\sum_{i=1}^{N}\left(a_{i}^{+}\right)^{l},
$$

where $k_{i}=0,1,2, \ldots$ are integral numbers (see, for instance, [22]) and the non-shifted staircase operators $a_{i}^{+}$(with $f=0$ ) are used. The corresponding spectrum coincides with the one of the original Calogero-oscllator model:

$$
E_{k_{1} \ldots k_{N}}=\frac{1}{2} N(g(N-1)+1)+\sum_{l=1}^{N} l k_{l} .
$$

Note that the fist term is just the ground-state energy.

\section{Non-Hermitian $P T$-invariant Hamiltonians}

Finally, consider the transformation (8), (9) with an imaginary phase. Keeping the old notation, we just make anywhere the substitution $f \rightarrow \imath f$ so that

$$
U=F^{-f}=\prod_{1 \leq i-j \leq r}\left|x_{i}-x_{j}\right|^{-f} .
$$

This is not an unitary map any more so the shifted Hamiltonians become now non-Hermitian. But they, of course, preserve the spectrum. In addition, they remain invariant under the simultaneous application of the parity transformation $P$ and time reversal $T$. The $P T$ transformation is characterized by the map $x_{i} \rightarrow-x_{i}$ and $\iota \rightarrow-l$. 
It is easy to see, in particular, that a non-Hermitian version of the system (10) is given by the Hamiltonian

$$
H=-\sum_{i=1}^{N} \frac{\partial_{i}^{2}}{2}-\sum_{1 \leq i-j \leq r}\left(\frac{f(f-1)}{\left(x_{i}-x_{j}\right)^{2}}-\frac{f}{x_{i}-x_{j}}\left(\partial_{i}-\partial_{j}\right)\right)+\sum_{\substack{i<j<k \\ r<k-i \leq 2 r}} \frac{f^{2}}{\left(x_{j}-x_{i}\right)\left(x_{i}-x_{k}\right)} .
$$

Note that the following term here spoils the Hermiticity but still obeys the $P T$ symmetry:

$$
\frac{f}{x_{i}-x_{j}}\left(\partial_{i}-\partial_{j}\right)
$$

Note that recently complex deformations of integrable models, related especially to the Calogero-type models, which preserve both the (super)integrability and $P T$ symmetry, has been quite popular subject of investigation (see, for example, [24, 25] and references therein). Inspired by this activity, let us apply the same conjugation (23) also to the Calogero model (15) in order to arrive at its non-Hermitian extension:

$$
\begin{aligned}
H_{g}= & \sum_{i} \frac{p_{i}^{2}}{2}+\sum_{1 \leq i-j \leq r}\left(\frac{g(g-1)-f(f-1)}{\left(x_{i}-x_{j}\right)^{2}}+\frac{f}{x_{i}-x_{j}}\left(\partial_{i}-\partial_{j}\right)\right)+\sum_{i-j>r} \frac{g(g-1)}{\left(x_{i}-x_{j}\right)^{2}} \\
& +\sum_{\substack{i<j<k \\
r<k-i \leq 2 r}} \frac{f^{2}}{\left(x_{i}-x_{j}\right)\left(x_{j}-x_{k}\right)} .
\end{aligned}
$$

For $r=N-1$ case when the distance of interactions is not restricted, the above model simplifies drastically. It is reduced to the Calogero model with modified coupling and additional terms (25) linear in momentum [23]:

$$
H_{g}=\sum_{i} \frac{p_{i}^{2}}{2}+\sum_{i<j}\left(\frac{g(g-1)-f(f-1)}{\left(x_{i}-x_{j}\right)^{2}}+\frac{f}{x_{i}-x_{j}}\left(\partial_{i}-\partial_{j}\right)\right) .
$$

In case of an equal value of both couplings, $f=g$, the inverse-square interaction disappears, and system is reduced to the gauged Calogero Hamiltonian, which may be considered as a Laplace operator deformed by a terms which are linear in momentum,

$$
H_{f}=-\frac{1}{2} \sum_{i} \partial_{i}^{2}+\sum_{i<j} \frac{f}{x_{i}-x_{j}}\left(\partial_{i}-\partial_{j}\right)
$$

\section{Acknowledgments}

S. V. acknowledges a support from the Regional Doctoral Program on Theoretical and Experimental Particle Physics Program sponsored by VolkswagenStiftung. T. H. was supported by the Armenian Committee of Science Grants No. 20TTAT-QTa009 and No. 20TTWS-1C035. The work was fulfilled within the ICTP Affiliated Center Program AF-04 and ICTP Network project NT-04. 


\section{References}

[1] F. Calogero, Solution of a three-body problem in one dimension, J. Math. Phys. 10 (1969) 2191; Solution of the one-dimensional $N$-body problems with quadratic and/or inversely quadratic pair potentials, ibid. 12 (1971) 419.

[2] J. Moser, Three integrable Hamiltonian systems connected with isospectral deformations, Adv. Math. 16 (1975) 197.

[3] M. A. Olshanetsky and A. M. Perelomov, Classical integrable finite dimensional systems related to Lie algebras, Phys. Rept. 71 (1981) 313; Quantum integrable systems related to Lie algebras, ibid. 94 (1983) 313.

[4] A. P. Polychronakos, Physics and mathematics of Calogero particles, J. Phys. A 39 (2006) 12793 [hep-th/0607033].

[5] A. Khare, Exact solution of an N-body problem in one dimension, J. Phys. A 29 (1996) L45; P. K. Ghosh and A. Khare, Relationship between the energy egenstates of CalogeroSutherland models with oscillator and Coulomb-like potentials, J. Phys. A 32 (1999) 2129 [solv-int/9808005].

[6] S. Wojciechowski, Superintegrability of the Calogero-Moser system, Phys. Lett. A 95 (1983) 279.

[7] V. B. Kuznetsov, Hidden symmetry of the quantum Calogero-Moser system, Phys. Lett. A 218 (1996) 212 [solv-int/9509001].

[8] T. Hakobyan, O. Lechtenfeld, and A. Nersessian, Superintegrability of generalized Calogero models with oscillator or Coulomb potential, Phys. Rev. D 90 (2014) 101701(R) [arXiv: 1409. 8288].

[9] T. Hakobyan and A. Nersessian, Runge-Lenz vector in Calogero-Coulomb problem, Phys. Rev. A 92 (2015) 022111 [arXiv: 1504.00760].

[10] A. Polychronakos, Exchange operator formalism for integrable systems of particles, Phys. Rev. Lett. 69 (1992) 703 [hep-th/9202057].

[11] L. Brink, T. Hansson, and M. Vasiliev, Explicit solution to the N-body Calogero problem, Phys. Lett. B 286 (1992) 109 [hep-th/9206049].

[12] C. F. Dunkl, Differential-difference operators associated to reflection groups, Trans. Amer. Math. Soc. 311 (1989) 167.

[13] A. Turbiner, Hidden algebra of the N-body Calogero problem, Phys. Lett. B 320 (1994) 281 [hep-th/9310125].

[14] M. Feigin and T. Hakobyan, On the algebra of Dunkl angular momentum operators, JHEP 11 (2015) 107 [arXiv: 1409.2480]. 
[15] M. Feigin and T. Hakobyan, Algebra of Dunkl Laplace-Runge-Lenz vector [arXiv: 1907.06706].

[16] N. Gurappa, P. K. Panigrahi, Equivalence of the Calogero-Sutherland model to free harmonic oscillators, Phys. Rev. B 59 (1999) R2490-R2493 [cond-mat/9710035].

[17] S. M. Pittman, M. Beau, M. Olshanii, A. del Campo, Truncated Calogero-Sutherland models, Phys. Rev. B 95, 205135 (2017) [arXiv: 1606.00895].

[18] F. Correa, T. Hakobyan, O. Lechtenfeld, and A. Nersessian, Spherical Calogero model with oscillator/ Coulomb potential: classical case, Phys. Rev. D 93 (2016) 125008 [arXiv: 1604.00026]; Spherical Calogero model with oscillator/Coulomb potential: quantum case, Phys. Rev. D 93 (2016) 125009 [arXiv: 1604 . 00027].

[19] T. Hakobyan, O. Lechtenfeld, A. Nersessian, The spherical sector of the Calogero model as a reduced matrix model, Nucl. Phys. B 858 250-266 (2012) [arXiv: 1110.5352].

[20] B. Basu-Mallick, A. Kundu, Exact solution of a many body problem with nearest and nextnearest neighbour interactions, Phys. Lett. A 279 (2001) 29-32 [cond-mat/0008199].

[21] A. Polychronakos, Non-relativistic bosonization and fractional statistics, Nucl. Phys. B 324 (1989) 597-622; T. Brzezinski, C. Gonera, P. Maslanka, On the equivalence of the rational Calogero-Moser system to free particles, Phys. Lett. A 254 (1999) 185-196 [hep-th/9810176].

[22] M. Feigin, O. Lechtenfeld, and A. Polychronakos, The quantum angular Calogero-Moser model, JHEP 1307 (2013) 162 [arXiv: 1305 . 5841].

[23] B. Basu-Mallick, A. Kundu, Exact solution of Calogero model with competing long-range interactions, Phys. Rev. B 62 (2000) 9927 [cond-mat/0003425]; B. Basu-Mallick, Fractional statistics in some exactly solvable Calogero-like models with PT-invariant interactions, Int. J. Mod. Phys. B 16 (2002) 1875-1882 [cond-mat/0201074].

[24] A. Fring, Particles versus fields in PT-symmetrically deformed integrable systems, Pramana 73 (2009) 363-374 [arXiv:0903.4670]; A note on the integrability of non-Hermitian extensions of Calogero-Moser-Sutherland models, Mod. Phys. Lett. A 21 (2006) 691-699 [hep-th/0511097].

[25] F. Correa, O. Lechtenfeld, PT deformation of angular Calogero models, JHEP 1711 (2017) 122 [arXiv: 1705.05425$]$. 